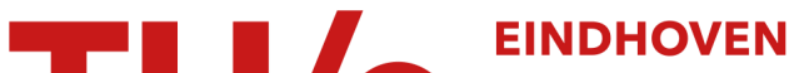 UNIVERSITY OF TECHNOLOGY
}

\section{Influence of buoyancy on drainage of a fractal porous medium}

Citation for published version (APA):

Huinink, H. P., \& Michels, M. A. J. (2002). Influence of buoyancy on drainage of a fractal porous medium. Physical Review E - Statistical, Nonlinear, and Soft Matter Physics, 66(4), 046301-1/6. [046301]. https://doi.org/10.1103/PhysRevE.66.046301

DOI:

10.1103/PhysRevE.66.046301

Document status and date:

Published: 01/01/2002

\section{Document Version:}

Publisher's PDF, also known as Version of Record (includes final page, issue and volume numbers)

\section{Please check the document version of this publication:}

- A submitted manuscript is the version of the article upon submission and before peer-review. There can be important differences between the submitted version and the official published version of record. People interested in the research are advised to contact the author for the final version of the publication, or visit the $\mathrm{DOI}$ to the publisher's website.

- The final author version and the galley proof are versions of the publication after peer review.

- The final published version features the final layout of the paper including the volume, issue and page numbers.

Link to publication

\section{General rights}

Copyright and moral rights for the publications made accessible in the public portal are retained by the authors and/or other copyright owners and it is a condition of accessing publications that users recognise and abide by the legal requirements associated with these rights.

- Users may download and print one copy of any publication from the public portal for the purpose of private study or research.

- You may not further distribute the material or use it for any profit-making activity or commercial gain

- You may freely distribute the URL identifying the publication in the public portal.

If the publication is distributed under the terms of Article 25fa of the Dutch Copyright Act, indicated by the "Taverne" license above, please follow below link for the End User Agreement:

www.tue.nl/taverne

Take down policy

If you believe that this document breaches copyright please contact us at:

openaccess@tue.nl

providing details and we will investigate your claim. 


\title{
Influence of buoyancy on drainage of a fractal porous medium
}

\author{
H. P. Huinink* and M. A. J. Michels \\ Department of Applied Physics, Technische Universiteit Eindhoven, P.O. Box 513, 5600 MB Eindhoven, The Netherlands
}

(Received 4 February 2002; published 1 October 2002)

\begin{abstract}
The influence of stabilizing hydrostatic pressure gradients on the drainage of a fractal porous medium is studied. The invasion process is treated with invasion percolation (IP) in a gradient. Fractality is mimicked by randomly closing bonds of a network. Two length scales govern the problem: the characteristic length of the pore structure $\xi_{s}$ and a length scale $\xi_{g}$ above which buoyancy determines the structure of the cluster. When $\xi_{s}<\xi_{g}$ the local structure of the invading cluster is governed by the interplay of capillarity and the fractal properties of the pore space. Only parts of the backbone of the pore structure can be invaded. Therefore, the obtained fractal dimension for small systems $L<\xi_{s}$ is much lower (1.40) than the one for ordinary IP (1.82). On larger length scales, $\xi_{s}<L<\xi_{g}$, the fractality of the pore space is no longer important and the cluster grows as in ordinary IP. When $L>\xi_{g}$, gravity becomes important and $\xi_{g}$ scales with the bond number $B$ as $\xi_{g}$ $\propto B^{-0.57}$, as in ordinary IP, while the fractal dimension becomes equal to the Euclidean one. When $\xi_{g}<\xi_{s}$ gravity is already important on length scales where the fractality of the medium has to be considered too. On small scales $L<\xi_{g}$, where only capillarity and fractality play a role the cluster structure is again characterized by the fractal dimension of 1.40 . On larger length scales, $\xi_{g}<L<\xi_{s}$, gravity promotes a more efficient invasion of the pore space and a different fractal dimension of 1.52 is found. The length scale $\xi_{g}$ no longer follows ordinary IP scaling: $\xi_{g} \propto B^{-0.69}$. When $L>\xi_{s}$ the fractal dimension of the invading cluster equals the Euclidean one and $\xi_{g} \propto B^{-0.69}$.
\end{abstract}

DOI: 10.1103/PhysRevE.66.046301

PACS number(s): 47.53.+n, 47.55.Mh, 64.60.Ak

\section{INTRODUCTION}

Multiphase-flow phenomena in porous media play an important role in many industrial and environmental processes [1]. The flow properties of an oil-water mixture through a specific porous rock determine the fraction of oil that can be obtained from this rock. Drying processes influence the durability of construction materials. Moisture transport in soil is an important aspect of many agricultural issues. In all these processes the geometry of the pore space is one of the parameters that determines the transport properties. Generally, the porous medium cannot be regarded as homogeneous below a certain length scale [2] and the geometry of the pore space may have fractal properties [3]. For instance, the pore spaces of sandstones are fractal and are self-similar over a few orders of magnitude in length, varying from $10 \AA$ to 100 $\mu \mathrm{m}$ [4]. The pore space of a widely used construction material such as concrete cannot be regarded to be homogeneous on length scales of the order of a few centimeters. Concrete contains so-called aggregate particles, which are impermeable and range in size from $100 \mu \mathrm{m}$ up to $1 \mathrm{~cm}$. In this study, we are interested in multiphase-flow phenomena on length scales where the medium is not homogeneous anymore.

The application of percolation models has improved our knowledge of the microscopic origins of multiphase-flow phenomena such as drainage, imbibition, and drying [5-7]. In the majority of these percolation studies, the porous medium is represented as a regular network of pores (sites) connected by throats (bonds). The disorder of the system is mimicked by varying the sizes of the pores and the throats.

\footnotetext{
*Corresponding author.

FAX: + +3140244 5253. Email address: h.p.huinink@tue.nl
}

Two different developments were of great importance. First, since the introduction of the invasion-percolation (IP) scheme our understanding of the role of capillarity on the structure of liquid clusters has greatly improved [8-10]. In IP a cluster grows by invading pores adjacent to the surface of the cluster. In drainage, which is the phenomenon of interest in this paper, the pore with the lowest pressure threshold $P=\gamma / r$ is invaded ( $\gamma$ and $r$ are the surface tension and throat radius respectively). This means that the pore with the widest throat is selected. Second, gradient percolation (GP) $[11,12]$ has provided tools for studying the influence of pressure gradients induced by buoyancy or viscous forces. By combining these two techniques the influence of buoyancy and viscosity on the structure of invading clusters, and in particular the width of the invasion front, could be studied [13-17]. Recently, this has been generalized to situations where the pressure gradients act both in the direction parallel and perpendicular to the flow direction [18].

As mentioned before, in the majority of these percolation studies, the porous medium is represented as a regular network of pores (sites) connected by throats (bonds). As a consequence the characteristic length $\xi_{s}$ of the medium always equals the distance between two neighboring lattice points $l$. Hence, fractal pore structures or heterogeneities on length scales larger than the average pore size cannot be accounted for. A way to mimic fractality in network models is by closing randomly bonds or sites. The structure of the pore space is in this approach the same as the structure of a samplespanning cluster in ordinary percolation (OP). This has been done in a few studies on invasion percolation $[19,20]$ and will be done in this study too. It was found that the fractal dimension $D$ of the invading cluster becomes dependent on the upper length scale of interest, $L$. When $L$ is larger than the correlation length $\xi_{s}$ the value $D=1.8$ is found for a two- 
dimensional (2D) system [21], which is the value for IP with trapping already in nonfractal geometries. In the case where $L<\xi_{s}$, it was found that the value of the fractal dimension is much smaller, caused by the fact that dead ends in the porous system cannot be invaded. Another interesting way to generate long-range structured porous network with fractal properties is by a fractional Brownian motion (fBM) [22-25]. This proves relevant for flow through fractured porous rock as encountered in oil recovery. In contrast to the common fractal porous geometries studied in this paper, such rock formations may have long-range structural correlations that increase with distance and that can be described by a socalled Hurst exponent $H$. Above the cutoff length of the correlations (above $\xi_{s}$ ) normal IP behavior is found with $D$ $=1.8$. Below this cutoff length the structure of the invading cluster varies with the Hurst exponent $H$. The cluster structure changes from fractal to compact, when the $H$ becomes larger then 0.5 .

When the porous medium has a long-range fractal structure, then fractality may play role on length scales where pressure gradients become important. In this particular study, we want to investigate the influence of gradients in the hydrostatic pressure (buoyancy) on the drainage of a 2D porous network with fractal properties. We limit ourselves to stabilizing gradients. Fractality is introduced by randomly closing a fraction $1-p$ of all bonds (OP). In Sec. II we subsequently discuss the model, the various existing regimes, and scaling results for these regimes. In Sec. III, results of IP simulations will be presented to investigate some unknown features of the scaling results. Finally, in Sec. IV the conclusions are drawn.

\section{THEORY}

\section{A. Model}

The pore space is mimicked by a square lattice with grid spacing $l$. The nodes of the network represent the pores and the bonds represent the throats. We assume that the volume of the pore system is in the pores and the resistance results from the throats. Fractality is generated by randomly closing a fraction $1-p$ of all bonds. Sites that are not connected with either the top or the bottom of the network via a string of open bonds are closed too, because they are considered to be a part of the solid matrix. Radii $r$ are assigned to the open throats according to a distribution function $\alpha(r)$. In this particular study, we use a uniform distribution with an average radius $\bar{r}$ and a width $\lambda$.

Initially all open sites are filled with the wetting $(w)$ fluid. The nonwetting (nw) fluid invades from the bottom and the $w$ fluid escapes from the top. The invasion algorithm consists of two fundamental steps. First, all interface sites that can be invaded are identified. These sites are the sites filled with $w$ fluid, which have neighboring sites filled with nw fluid and do not belong to a trapped cluster. Second, the interface site with the lowest invasion potential $Q$ is selected and invaded. The invasion potential $Q_{i j}$ of an interface site $i$ invaded from a site $j$ is

$$
Q_{i j}=\frac{\gamma}{r_{i j}}+\Delta \rho g z_{i}=\frac{\gamma}{l}\left(\frac{1}{\widetilde{r}_{i j}}+B \widetilde{z_{i}}\right),
$$

where $\gamma$ is the surface tension, $\Delta \rho$ the density difference between the nw and $w$ fluid $\left(\Delta \rho \equiv \rho_{\mathrm{nw}}-\rho_{w}\right), g$ the gravity acceleration, $r_{i j}$ the radius of the throat between the sites $i$ and $j\left(\widetilde{r}_{i j} \equiv r_{i j} / l\right), z_{i}$ the height of the pore $i\left(\widetilde{z}_{i} \equiv z_{i} / l\right)$, and $B$ is the bond number

$$
B \equiv \frac{\Delta \rho g l^{2}}{\gamma}
$$

which is the ratio of the typical buoyancy forces and capillary forces on the pore level. It follows from Eq. (1) that two different situations can be distinguished when the nw fluid invades from the bottom of the system: (a) the nw fluid is heavier than the $w$ fluid $(B>0)$ and $(b)$ the opposite situation $(B<0)$. When $B>0$ sites with low $\widetilde{z}_{i}$ are preferentially invaded and a stable front develops. This is called invasion percolation in a stabilizing gradient. When $B<0$ the nw fluid preferentially invades sites with high $\widetilde{z}_{i}$ and fingering occurs, which is called invasion percolation in a destabilizing gradient. Because we want to study invasion processes in a stabilizing gradient due to gravity, we limit the remainder of the discussion to $B>0$.

\section{B. Regimes of length scales}

Two length scales will determine the behavior of the invading cluster: the correlation length of the porous network, which in OP has a universal dependence on the fraction $p$ of

$$
\xi_{s} \propto\left|p-p_{c}\right|^{-\nu}
$$

and a length scale related with the influence gravity

$$
\xi_{g} \propto|B|^{-\mu}
$$

Here $\nu$ is the well-known correlation-length exponent $(\nu$ $=4 / 3$ in $2 \mathrm{D}$ ) and $\mu$ is the exponent of interest. For IP on a regular network it was found that $\mu=\nu /(\nu+1)$ [13]. For the moment, we will assume that the general form of Eq. (4) also holds for fractal porous networks. In Sec. III, we will prove that this assumption is correct. It has to be remarked that the scaling laws only have a meaning for $\xi_{s}>l$ and $\xi_{g}>l$. With the length scales $L, \xi_{s}$ and $\xi_{g}$ a diagram can be constructed, see Fig. 1, which makes clear that five different regimes exist: (i) invasion percolation in a fractal medium (IPF), (ii) ordinary invasion percolation (IP), (iii) invasion percolation in a fractal medium in the presence of a gradient (IPFG), (iv) ordinary invasion percolation in a gradient (IPG), and (v) invasion percolation in a very strong gradient (IPGs). These regimes will be discussed below.

(i) The lower left part of Fig. 1 is the regime where capillary effects dominate the displacement processes and the structure has to be regarded as fractal $\left(L<\xi_{s}, \xi_{g}\right)$. We will call this IPF. It is known that in this regime the underlying structure of the pore network will influence the cluster structure $[20,25]$. We expect that the mass $M$ of the invading cluster scales in this regime with a fractal dimension $D_{0}$ 


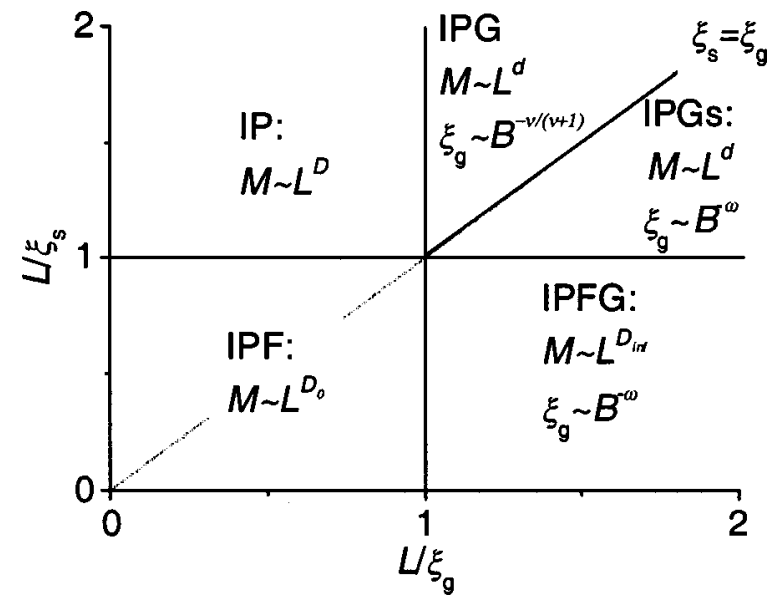

FIG. 1. A schematic overview of the different scaling regimes for the mass $M$ of the nonwetting cluster. The solid lines are the boundaries of the various regimes. In these regimes $M$ scales differently with the system size $L$. The correlation length $\xi_{g}$ scales differently with the bond number $B$.

considerably lower than in standard IP. Beforehand we know that its value will be in between the fractal dimensions of the minimal path and the backbone of the porous fractal network, $D_{\min }<D_{0}<D_{b}$, and therefore $1.13<D_{0}<1.62$ in $2 \mathrm{D}$ $[26,27]$, because the nw fluid can only invade the backbone sites of the pore space and the $w$ fluid will be trapped in the dead ends. It is already known from simulations that for fractal structures created via site percolation $D_{0}=1.37$ [20].

(ii) The upper left part of the diagram is the regime where fractality is not important anymore, but the displacements are still dominated by capillary effects $\left(\xi_{s}<L<\xi_{g}\right)$. On larger length scales the structure of the invading cluster behaves as in standard IP and $D=1.82[21]$; the latter value has been found for fractal structures generated via site percolation [20]. This crossover to standard IP with trapping was also found for IP on lattices generated with fBM. Below the correlation length (cutoff length) the structure of the invading cluster strongly depends on the underlying structure of the lattice; but on length scales larger than the cutoff length the invading cluster is characterized by a fractal dimension $D$ $=1.82$ [25].

(iii) The lower right part of diagram, $\xi_{g}<L<\xi_{s}$, corresponds to IPFG. Gravity significantly influences the displacements process. The structure of the medium has still to be regarded as fractal. We expect that the value of the fractal dimension $D_{\text {inf }}$ will be in between the values for the IPF cluster and the backbone of the pore space $\left(D_{0}<D_{\text {inf }}\right.$ $<D_{b}$ ). As in the IPF regime the invading cluster will only grow in the backbone of the porous structure, but in a more efficient way than in the absence of gradients. Another interesting point of this regime is the behavior of the correlation length $\xi_{g}$. We assume that in this regime Eq. (4) applies, which will prove to be correct in Sec. III. In that section we also calculate the value of $\mu$ in this regime and show that the value $\omega$ for the exponent $\mu$ satisfies $\mu=\omega \neq \nu /(\nu+1)$.

(iv) The upper triangle in the upper right part of Fig. 1 is the regime where gravity only influences the invasion process on length scales much larger than the correlation length of the pore structure $\left(\xi_{s}<\xi_{g}<L\right)$. Therefore, the invading cluster behaves as in ordinary IPG and a front will develop of which the width $\sigma$ scales proportional to $\xi_{g}$. The exponent $\mu$ is therefore $\nu /(\nu+1)$, which is $4 / 7$ in $2 \mathrm{D}[13,14]$. On large length scales the cluster is not fractal anymore and its mass scales with $d$ (the dimension of the system, which is two in our case).

(v) The last regime we have to discuss is the one represented by the lower triangle in the upper right part of the diagram. In this regime fractality is not important on large length scales. However, gravity is important on length scales on which the medium has to be regarded as fractal $\left(\xi_{g}<\xi_{s}\right.$ $<L)$. We will call this IPGs. As in IPG a front develops. We expect that width of this front is of the order of $\xi_{s}$ and that the gradient only influences the finer details of the front. The correlation length $\xi_{g}$ will behave as in the IPFG regime. The mass of the invading cluster will scale with the system dimension $d$.

\section{Scaling relations}

Figure 1 suggests that it should be possible to find a scaling relation for the mass of the invading cluster, which should have the following form:

$$
M\left(\frac{1}{\xi_{g}}, \frac{L}{\xi_{s}}\right)=A L^{D_{0}} f\left(\frac{L}{\xi_{g}}, \frac{L}{\xi_{s}}\right),
$$

where $A$ is a constant and $f$ the scaling function. By combination of the scaling relations shown in Fig. 1 and using $\xi_{s}$ $=L, \xi_{g}=L$, and $\xi_{s}=\xi_{g}$ at the boundaries of the various regimes (the solid lines), we arrive at

$$
M=A L^{D_{0}} \begin{cases}x^{d-D_{0}}\left(\frac{y}{x}\right)^{D-D_{0}}, & x<y \\ y^{d-D_{0}\left(\frac{x}{y}\right)^{D_{\mathrm{inf}}-D_{0}},}, & x>y,\end{cases}
$$

where

$$
x=\left\{\begin{array}{ll}
L / \xi_{g}, & \xi_{g}<L \\
1, & \xi_{g}>L
\end{array} \text { and } y= \begin{cases}L / \xi_{s}, & \xi_{s}<L \\
1, & \xi_{s}>L\end{cases}\right.
$$

It follows from Eq. (7) that $x<y$ refers to IP $\left(\xi_{g}>L\right)$ and IPG $\left(\xi_{g}<L\right)$ regimes, where $\xi_{s}<\xi_{g}$ (see Fig. 1). In this particular case gravity does not play a role on length scales at which the fractal properties of the medium have to be taken into account. When $x>y$, which means that the system is either in the IPFG $\left(\xi_{g}>L\right)$ or IPFs $\left(\xi_{g}<L\right)$ regime $\left(\xi_{s}\right.$ $>\xi_{g}$ ), the influence of gravity cannot be neglected on length scales where the fractal properties of the medium have to be considered explicitly. This becomes visible in Eq. (6) by the entering of the fractal dimension $D_{\text {inf }}$. The point $x=y=1$ corresponds with the IPF regime.

From our discussion of Fig. 1, it follows that the regimes IPF and IPFG $\left(\xi_{s}>L\right)$ are the unknown and therefore interesting parts of the phase diagram. For these particular regimes formula (6) can be written as 


$$
M=A^{\prime} L^{D_{0}}\left\{\begin{array}{lll}
\left(L B^{\mu}\right)^{D_{\text {inf }}-D_{0}}, & \xi_{g}<L & (\mathrm{IPFG}) \\
1, & \xi_{g}>L & (\mathrm{IPF}),
\end{array}\right.
$$

where $A^{\prime}$ is a constant and Eq. (4) is used. When $\xi_{g}<L$, the mass of the invading cluster depends on the bond number $B$. Because $D_{0}<D_{\text {inf }}$ and $\mu>0$, it follows from Eq. (8) that the mass of the invading cluster increases with $B$. So, as in IPG, gravity in this regime promotes a more efficient invasion of the pore structure by the nw fluid. The question still is to what extent this effect is modified by the fractality of the pore network.

\section{IP SIMULATIONS}

In the preceding section, we have discussed scaling formulas that describe the influences of gravity and of the fractality of the pore space on the structure of the invading cluster. However, the discussion of the IPF and IPFG is far from conclusive. First, it is assumed that on length scales smaller than $\xi_{s}$ the invading cluster has a fractal structure and can be described with two fractal dimensions: $D_{0}(B=0)$ and $D_{\text {inf }}$ $(B=\infty)$. Second, we have assumed that the crossover from $B=0$ to $B=\infty$ behavior can be described in the same way as it is done for $\xi_{g}>\xi_{s}$ by one length scale $\xi_{g}$ [Eq. (4)]. Third, the values of the introduced exponents $D_{0}, D_{\text {inf }}$, and $\mu$ are unknown. In this section, we want to address these issues by discussing IP simulations.

Simulations have been performed according to the model described in Sec. II A. All calculations are done on square lattices of $L \times 2 L$. Periodic boundary conditions are used for the long sides $(2 L)$ of the network. Properties are always calculated in the central $L \times L$ part of the system to exclude boundary effects. To avoid unnecessary computations, all simulations are done at $p=p_{c}$ and therefore $\xi_{s}=L$. We have distributed the throat radii according to a uniform distribution that has a width $\lambda / l=0.2$ and an average radius $\bar{r} / l$ $=0.15$. Every data point is an average of about 300 realizations.

First, we have calculated the mass of the invading cluster in the central region as a function of the system size for $B$ $=[0,100]$. In Fig. 2 we have plotted the mass per unit "volume," i.e., the saturation $M L^{-d}$ as a function of $L$ for $B$ $=0,0.01,0.1$, and 100. By fitting the data points corresponding with $B=0$ (dashed line) and $B=100$ (upper solid line), we have obtained $D_{0}=1.397 \pm 0.004$ and $D_{\text {inf }}=1.519$ \pm 0.005 . The value of $D_{0}$ is close to the value obtained in calculations where site percolation is used to introduce fractality in the medium [20]. To clarify the different scaling behavior of the two situations, $B=0$ and $B=100$, we have visualized both the nw phase and $w$ phase for a $L=100$ system, see Fig. 3. It can be seen that both the degree of branching becomes higher and the branches grow longer when gravity becomes important on the length scale of interest. This indicates that the loops in the backbone of the pore space are invaded more efficiently in the presence of pressure gradients due to gravity, resulting in a higher fractal

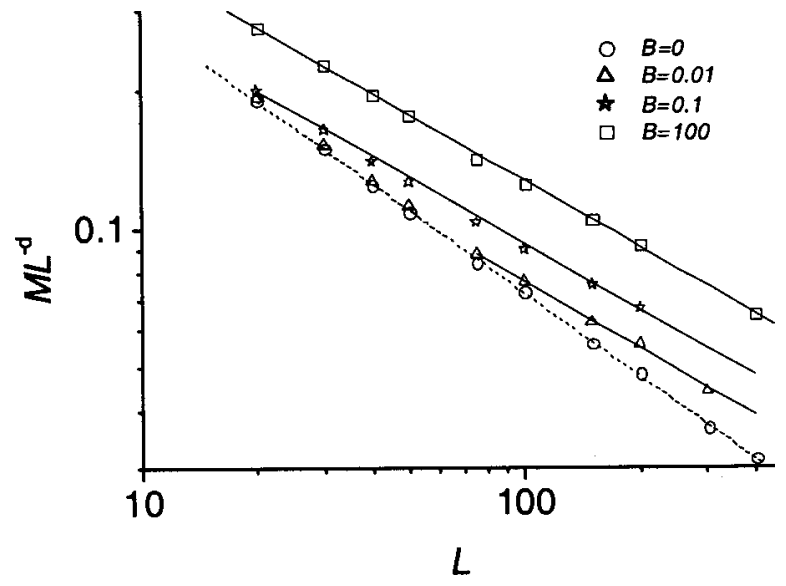

FIG. 2. The saturation of the system by the invading cluster of nw fluid, $M L^{-d}$. The upper solid line $\left(B=100, M L^{-d}\right.$ $\left.\propto L^{-0.481 \pm 0.005}\right)$ and the dashed line $\left(B=0, M L^{-d} \propto L^{-0.603 \pm 0.004}\right)$ are fitting results. All solid lines obey the same scaling law.

dimension. As expected, we have found that $D_{\min }<D_{0}$ $<D_{\text {inf }}<D_{b}$. That $D_{\text {inf }}<D_{b}$ can be understood as follows. Consider a loop consisting of two strings $I$ and $J$ of sites and bonds that have maximum heights $z_{I}^{\max }<z_{J}^{\max }$, see Fig. 4. At $B=\infty$ both strings are invaded such that nw/w interfaces in these strings are always at the same height. Therefore, the maximum height reached in both strings always equals $z_{I}^{\max }$. As a consequence $w$ fluid is trapped in that part of string $J$ lying between the point where $z=z_{I}^{\max }$ and the point where $I$ and $J$ come together, and the mass of the nw cluster is reduced.

For intermediate values of $B$ two different scaling regimes can indeed be distinguished, as proposed in the preceding section; this is seen in Fig. 2. For small $L$ all curves coincide and $M \propto L^{D_{0}}$. This indicates that gravity has no influence on the structure of the invading cluster on such length scales. Above a certain value of $L\left(=\xi_{g}\right)$ the cluster grows as in the
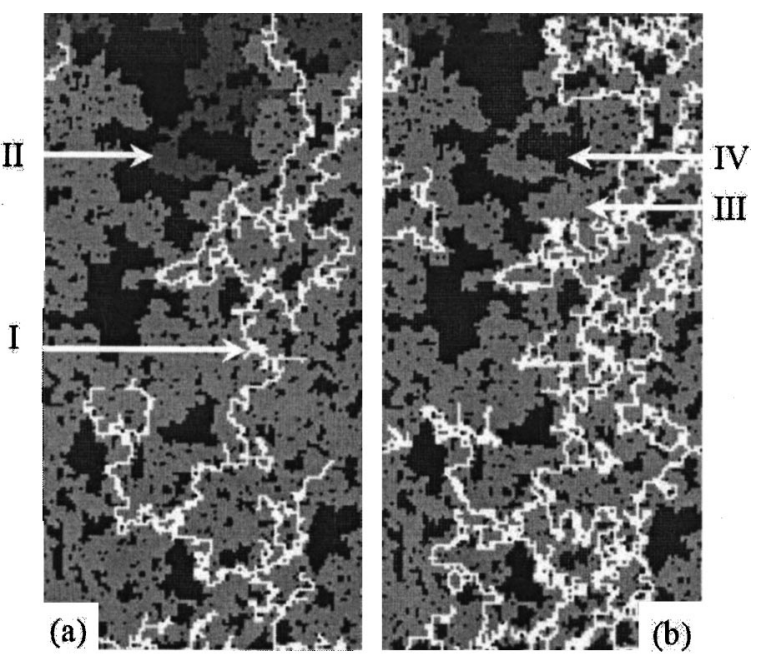

FIG. 3. The invading cluster at breakthrough in an $L=100$ system: (a) $B=0$ and (b) $B=100$. In this picture I refers to pores filled with nw fluid, II to pores filled with nontrapped $w$ fluid, III to pores that contain trapped $w$ fluid, and IV to the solid phase. 


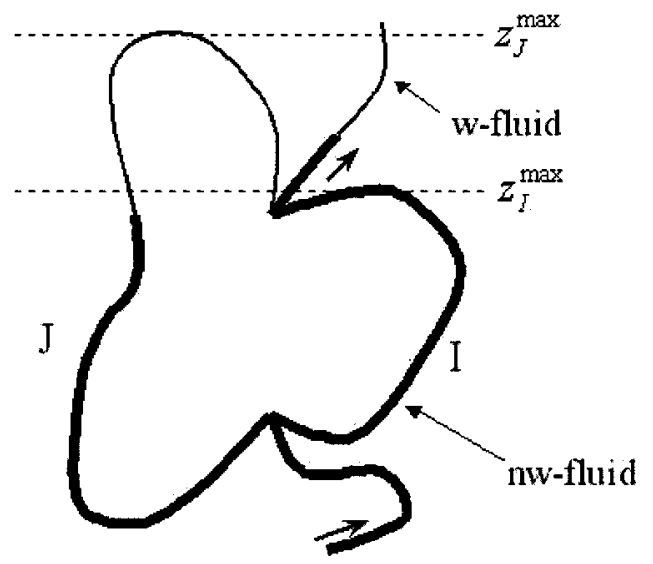

FIG. 4. The invasion of a loop by a nw fluid in the presence of a strong buoyancy force, $B=\infty$. The arrows mark the flow direction. Because a fraction of the $w$ fluid is trapped in the upper part of the loop, the fractal dimension of the nw cluster will be lower than the fractal dimension of the backbone of the pore space.

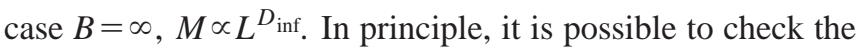
validity of Eq. (4) and find the value of the exponent $\mu$ directly by plotting the crossover length as a function of $B$. However, for reasons of accuracy we choose a different approach. In Fig. 5, we have plotted $M L^{-D_{\text {inf }}}$ as a function of $B$. By using Eq. (8) and fitting the $L=200$ data for $B$ $=[0.01,3]$, we obtain $\mu=0.69 \pm 0.01$. This figure proves that the assumption made in Sec. II, to derive scaling formulas, that the expression (4) also holds for $\xi_{g}<\xi_{s}$, is correct. Furthermore, we can conclude that fractality significantly modifies the value of $\mu$. The background of this particular value is unclear to us, because we have not been able to derive Eq. (3) from microscopic arguments for the situation $\xi_{g}<\xi_{s}$.

Finally, we have plotted $M L^{-D_{0}}$ as a function of $L B^{\mu}$ in Fig. 6 for a variety of values of $L$ and $B$. We have used the previously found values $D_{0}=1.40$ and $\mu=0.69$. Most of the data points collapse on one single master curve. Deviations occur for high values of $L B^{\mu}$, where the correlation length

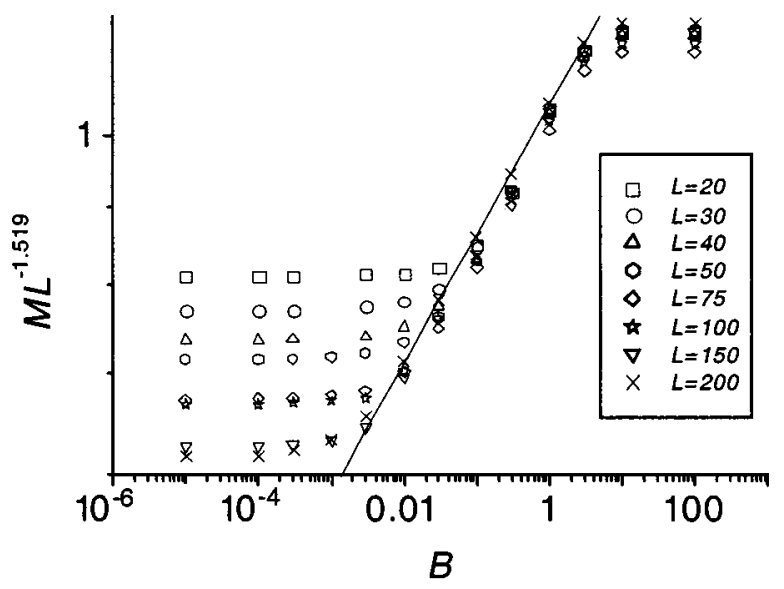

FIG. 5. Scaled mass as function of $B$ for different lattice sizes $L$. The solid line is a fit through the data points of $L=200$ for $B$ $=[0.01,3]$.

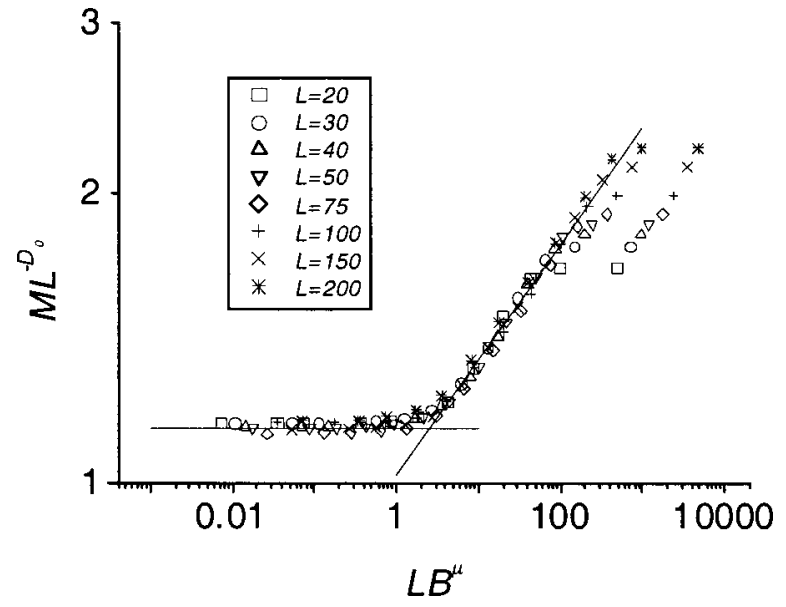

FIG. 6. The scaling function for the mass in the regime $\xi_{g}<\xi_{s}$.

saturates at the lowest possible value: the grid spacing $l$. We can conclude that Fig. 6 proves that the system indeed obeys scaling in the form of Eq. (8).

\section{CONCLUSIONS}

We have studied drainage of a fractal porous medium in the presence of a stabilizing hydrostatic pressure gradient. Three different length scales govern the problem: the system size $L$, the correlation length of the porous medium $\xi_{s}$, and a length scale $\xi_{g}$ above which gravity determines the structure of the fluid cluster.

When $\xi_{s}<\xi_{g}$, the large-scale $\left(L>\xi_{s}\right)$ behavior of the nonwetting liquid cluster is the same as in the case of ordinary invasion percolation in a gradient (with trapping): $M$ $\propto L^{1.82}$ and $\xi_{g} \propto B^{-0.57}$. Gravity then does not play a role in length scales where the fractality of the medium does play a role and vice versa. Deviations in the mass-size scaling occur on small length scales $\left(L<\xi_{s}\right)$, where the interplay of capillary forces and the fractality of the pore space is important. In this regime, we have obtained for the nonwetting cluster a fractal dimension $D_{0} \simeq 1.40$, which is caused by the fact that the dead ends of the pore space cannot be invaded by the nw fluid.

In the case that $\xi_{s}>\xi_{g}$ gravity is already important on length scales where the fractality of the medium has to be taken into account. At very small length scales $\left(L<\xi_{g}\right)$ the growth of the invading cluster can again be described with $D_{0}$. However, on larger length scales $\left(L>\xi_{g}\right)$ a larger fractal dimension has been found, $D_{\mathrm{inf}} \simeq 1.52$. Gravity promotes a more efficient invasion of the backbone of the pore space. The correlation length now scales as $\xi_{g} \propto B^{-0.69}$. More work has to be done, to understand the value of the exponent $\mu$ in this regime.

\section{ACKNOWLEDGMENT}

This project was financially supported by the Dutch Technology Foundation (STW). 
[1] F. A. L. Dullien, Porous Media (Academic, New York, 1979), pp. 343-347.

[2] J. Bear, Dynamics of Fluids in Porous Media (Elsevier, New York, 1972), pp. 15-21.

[3] A. H. Thompson, A. J. Katz, and C. E. Krohn, Adv. Phys. 36, 625 (1987).

[4] A. J. Katz and A. H. Thompson, Phys. Rev. Lett. 54, 1325 (1985).

[5] D. Stauffer and A. Aharony, Introduction to Percolation Theory, 2nd ed. (Taylor and Francis, London, 1992).

[6] M. Sahimi, Flow and Transport in Porous Media and Fractured Rock (VCH, Weinheim, 1995).

[7] P. Meakin, Fractals, Scaling and Growth Far from Equilibrium (Cambridge University Press, Cambridge, 1998).

[8] R. Lenormand and S. Bories, C. R. Acad. Sci., Paris B 291, 279 (1980).

[9] R. Chandler, J. Koplik, K. Lerman, and J. F. Willemsen, J. Fluid Mech. 119, 24 (1982).

[10] D. Wilkinson and J. F. Willemsen, J. Phys. A 18, 3365 (1983).

[11] B. Sapoval, M. Rosso, and J. F. Gouyet, J. Phys. (France) Lett. 46, L149 (1985).

[12] M. Rosso, J. F. Gouyet, and B. Sapoval, Phys. Rev. Lett. 57, 3195 (1986).
[13] D. Wilkinson, Phys. Rev. A 34, 1380 (1986).

[14] A. Birovljev, L. Furuberg, J. Feder, T. Jøssang, K. J. Maløy, and A. Aharony, Phys. Rev. Lett. 67, 584 (1991).

[15] V. Frette, J. Feder, T. Jøssang, and P. Meakin, Phys. Rev. Lett. 68, 3164 (1992).

[16] M. Chaouche, N. Rakotomalala, D. Salin, B. Xu, and Y. C. Yortsos, Phys. Rev. E 49, 4133 (1994).

[17] Y. C. Yortsos, B. Xu, and D. Salin, Phys. Rev. Lett. 79, 4581 (1997).

[18] Y. Zhang, M. Shariati, and Y. C. Yortsos, Transp. Porous Media 38, 117 (2000).

[19] U. Oxaal, M. Murat, F. Boger, A. Aharony, J. Feder, and T. Jøssang, Nature (London) 329, 33 (1987).

[20] R. Paredes and M. Octavio, Phys. Rev. A 46, 994 (1992).

[21] M. M. Dias and D. Wilkinson, J. Phys. A 19, 3131 (1986).

[22] M. B. Isichenko, Rev. Mod. Phys. 64, 961 (1992).

[23] M. Sahimi, J. Phys. I 4, 1263 (1994).

[24] C. Du, C. Satik, and Y. C. Yortsos, AIChE J. 42, 2392 (1996).

[25] M. A. Knackstedt, M. Sahimi, and A. P. Sheppard, Phys. Rev. E 61, 4920 (2000).

[26] H. J. Hermann and H. E. Stanley, Phys. Rev. Lett. 53, 1121 (1984).

[27] H. J. Hermann and H. E. Stanley, J. Phys. A 21, L829 (1988). 